\title{
IMPACTO DE FALLAS EN LAS BUENAS PRÁCTICAS DE MANUFACTURA EN LA PRODUCCIÓN DE CARNE MOLIDA DE RES EN CARNICERÍAS DE TRES MUNICIPIOS DE LA ZONA METROPOLITANA DE GUADALAJARA
}

\author{
Impact of failure in good manufacturing practices during the production of ground beef \\ in butcher's shops at three Municipalities of the Metropolitan Area of Guadalajara
}

Elisa Cabrera Díaz ${ }^{1 *}$, Andrea Iliana Ascencio Anguiano ${ }^{1}$, Luis Eduardo Segura García ${ }^{1}$, Olga Berenice Pérez Covarrubias ${ }^{1}$, Adriana Guadalupe Orozco García ${ }^{1}$, Juan José Varela Hernández ${ }^{2}$, Gustavo González González ${ }^{3}$

${ }^{1}$ Departamento de Salud Pública. Centro Universitario de Ciencias Biológicas y Agropecuarias. Universidad de Guadalajara, Zapopan, Jalisco, México. ${ }^{2}$ Departamento de Ciencias Médicas y de la Vida, Centro Universitario de la Ciénega. Universidad de Guadalajara, Ocotlán, Jalisco, México. ${ }^{3} 3 \mathrm{M}$ FSD LATAM, Guadalajara, Jalisco, México

* Autor de correspondencia: elisa.cabrera@ academicos.udg.mx

\section{Resumen}

La carne molida de res insuficientemente cocinada genera casos y brotes de enfermedades entre ellas, salmonelosis. En México, la carne molida se fabrica a nivel industrial, y también en gran medida, en carnicerías locales donde el cumplimiento de las buenas prácticas de manufactura (BPM) suele ser deficiente. Para determinar la higiene del proceso de fabricación y frecuencia de aislamiento de Salmonella se analizaron 96 muestras de carne molida de res recolectadas en 96 carnicerías de tres municipios de la Zona Metropolitana de Guadalajara. En cada muestra se realizó el recuento de $E$. coli y coliformes totales utilizando placas $3 \mathrm{M}^{\mathrm{TM}}$ Petrifilm $^{\mathrm{TM}}$ y se detectó la presencia de Salmonella con el equipo de detección molecular 3M MDS con posterior confirmación por cultivo. Los niveles de coliformes oscilaron entre 2.2 y 7.4 $\log \mathrm{UFC} / \mathrm{g}$ con un promedio de $4.7 \pm 1.03 \mathrm{log}$ UFC/g, y los de E. coli entre 1.7 y $6.9 \mathrm{log}$ $\mathrm{UFC} / \mathrm{g}$ con promedio de $3.5 \pm 0.77 \mathrm{log}$ UFC/g. Salmonella se detectó en el $68.8 \%$ de las muestras, con una frecuencia de aislamiento del $23.9 \%$ para los municipios A y B, y del $21 \%$ para el municipio C. Los recuentos de $E$. coli y frecuencia de Salmonella no fueron diferentes entre las muestras de carnicerías de diferentes municipios $(p>0.05)$. El $36.3 \%$ de las muestras positivas a Salmonella presentó niveles de $E$. coli $\geq 5,000 \mathrm{UFC} / \mathrm{g}$. Las fallas en las BPM en las carnicerías resultó en la fabricación de carne molida de res con altas cargas microbianas y alta frecuencia de Salmonella, lo que conlleva un riesgo potencial para el consumidor. Es necesario mejorar la capacitación en BPM del personal que labora en carnicerías, así como intensificar la inspección oficial que obligue su cumplimiento por parte de las autoridades correspondientes.

Palabras clave: Carne molida, Salmonella, E. coli, inocuidad, higiene, BPM 


\section{Abstract}

Insufficiently cooked ground beef can produce individual cases and outbreaks of diseases including salmonellosis. In México, ground beef is produced in large-scale processing plants, and also a high volume is produced in local butcher's shops where compliance with the good manufacturing practices (GMP) is deficient. To determine the hygiene conditions during the fabrication process and the isolation frequency of Salmonella, we analyzed 96 samples of ground beef collected from 96 butcher's shops located in three municipalities of the metropolitan area of Guadalajara. For each sample, enumeration of $E$. coli and total coliforms was performed using $3 \mathrm{M}^{\mathrm{TM}}$ Petrifilm ${ }^{\mathrm{TM}}$ plates, and the presence of Salmonella was detected using the 3M MDS molecular detection system with further confirmation by culture. Coliform counts ranged from 2.2 to $7.4 \mathrm{log} \mathrm{UFC} / \mathrm{g}$ with a mean value of $4.7 \pm 1.03 \mathrm{log} \mathrm{UFC} / \mathrm{g}$, while E. coli counts ranged from 1.7 to $6.9 \mathrm{log} \mathrm{UFC} / \mathrm{g}$ and showed mean values of $3.5 \pm 0.77 \mathrm{log} \mathrm{UFC} / \mathrm{g}$. Salmonella was detected in $68.8 \%$ of samples; the frequency observed in samples from municipalities A and B was $23.9 \%$ and $21 \%$ for municipality $\mathrm{C}$. The frequency of Salmonella and the E. coli counts were not different among samples collected from the three municipalities $(p>0.05)$. From those samples that tested positive for Salmonella, $36.3 \%$ showed $E$. coli counts $\geq 5,000 \mathrm{CFU} / \mathrm{g}$. Failure to comply with the GMP in the butcher's shops resulted in the fabrication of ground beef with high microbial load and high frequency of Salmonella, originating a potential health risk of consumers. It is necessary to improve training in GMP among butcher's shops workers, and also to intensify official inspections by authorities to enforce their compliance.
Key words: Ground beef, Salmonella, E. coli, food safety, hygiene, GMP

\section{Introducción}

La carne y productos cárnicos son alimentos que frecuentemente se asocian como causa de casos y brotes de enfermedad tanto en México como en otros países. Los patógenos comúnmente vinculados a enfermedades transmitidas por alimentos cárnicos son Salmonella, Escherichia coli productora de toxina Shiga (STEC), Campylobacter y Listeria monocytogenes. En los Estados Unidos (EUA) se reportó que durante el periodo del 2009 al 2015 se detectaron 201 brotes de enfermedades atribuidos al consumo de carnes rojas, causando 4,654 enfermos (CDC 2018). En México se presentan más de 70 mil casos de salmonelosis cada año (Secretaría de Salud 2019), aunque no se cuenta con un sistema de vigilancia epidemiológica que permita determinar cuántos de estos casos son transmitidos por alimentos, y en particular por consumo de carne y productos cárnicos.

La contaminación de la carne inicia en el establecimiento de sacrificio y durante el faenado de animales de abasto. Las principales fuentes de contaminación son las vísceras y piel de animales faenados, a partir de los cuales la materia fecal se transfiere hacia la superficie de las canales llevando consigo patógenos entéricos (Cabrera Díaz et al. 2010). La prevalencia de Salmonella en el ganado bovino varía entre regiones geográficas y depende de factores como tipo de ganado, alimentación y manejo. En México se ha reportado que entre el 5.6 y el $81.2 \%$ de los bovinos presentados a sacrificio portan al patógeno en su tracto intestinal (Narvaez-Bravo et al. 2013; Ruiz et al. 2013; Zaidi et al. 2008) y el $92.4 \%$ en la piel (Narvaez-Bravo et al. 2013). Otras fuentes de contaminación hacia la carne en canal pueden incluir el agua de lavado, equipos y utensilios 
que se usan en el proceso de faenado si no han sido previamente lavados y sanitizados adecuadamente, así como los trabajadores cuando no siguen buenas prácticas de higiene; el aire que ingresa y circula por el establecimiento donde se producen $y$ almacenan las canales constituye un mecanismo que puede trasladar contaminación desde zonas sucias hacia zonas limpias donde se produce o almacenan las canales. La prevalencia de Salmonella en canales de bovino refrigeradas en rastros municipales de nuestro país se ha reportado entre el 14 y 18\% (Hernández-San Juan et al. 2007; Martínez-Chávez et al. 2015; PérezMontaño et al. 2012) mientras que en rastros Tipo Inspección Federal (TIF) donde se aplican tratamientos antimicrobianos se ha reportado en un 6\% (Narvaez-Bravo et al. 2013). Más adelante, en el proceso de fabricación de cortes y productos cárnicos, la carne puede continuar contaminándose principalmente a través del contacto con equipos y utensilios mal sanitizados.

La carne molida de res consumida insuficientemente cocinada es causa frecuente de casos y brotes de salmonelosis y de infección por STEC (CDC 2018). Este alimento es un producto cárnico fresco que se fabrica a partir de recortes secundarios de la canal que pasan por un molino o picadora, y durante su fabricación se expone a diversas fuentes de contaminación, principalmente el molino y todas las superficies con las que tienen contacto los recortes (materia prima) y la propia carne durante la molienda; la carga microbiana de los recortes utilizados para la fabricación de la carne molida puede ser un aporte de contaminación importante al producto final obtenido.

En nuestro país, la fabricación de carne molida se realiza a nivel industrial, pero también en gran medida, en los puntos de venta al consumidor, es decir, en carnicerías y supermercados. En las carnicerías, el cumplimiento de las BPM suele ser deficiente y se identifican múltiples fuentes de contaminación (Martínez-Chávez et al. 2015). En trabajos previos, se ha reportado una frecuencia de Salmonella en el 32 al $71 \%$ de muestras de carne molida de res recolectadas en carnicerías (Cabrera-Diaz et al. 2013; Martínez-Chávez et al. 2015; Nayarit-Ballesteros et al. 2016) y en el 6\% de muestras de supermercados (NayaritBallesteros et al. 2016). En este este trabajo el objetivo fue determinar el impacto de las fallas a las buenas prácticas de higiene en la producción de carne molida de res a través del recuento de indicadores microbianos y de la detección de Salmonella en el producto fabricado en carnicerías de tres municipios de la Zona Metropolitana de Guadalajara, Jalisco.

\section{Materiales y métodos}

\section{Recolección de muestras}

Se recolectaron 96 muestras de carne molida de res fabricada en 96 diferentes carnicerías ubicadas en tres municipios de la Zona Metropolitana de Guadalajara, Jalisco. Las muestras fueron recolectadas entre los meses de junio y julio de 2019, con apoyo de las oficinas de Inspección Sanitaria de los Ayuntamientos correspondientes. Inspectores oficiales de cada ayuntamiento fueron quienes seleccionaron aleatoriamente las carnicerías incluidas en el estudio (32 carnicerías/municipio) y recolectaron las muestras; de cada carnicería colectaron una muestra de $500 \mathrm{~g}$ de carne molida de res que fue colocada en bolsa estéril de polietileno. Las muestras fueron transportadas en hieleras con refrigerantes al Laboratorio de Inocuidad de Alimentos del Departamento de Salud Pública del CUCBA en donde se mantuvieron refrigeradas $y$ fueron analizadas en un máximo de $2 \mathrm{~h}$ posteriores a su llegada.

Recuento de indicadores microbianos de higiene 
Para el recuento de E. coli genérica y coliformes, se pesaron $10 \mathrm{~g}$ de cada muestra de carne molida de res en una bolsa estéril de polietileno, se agregaron $90 \mathrm{~mL}$ de diluyente de peptona al $0.1 \%$ (DIFCO, USA) y se homogeneizó en un BagMixer (Mod. 400 P, Interscience, France) por 1 min; a partir de esta dilución se realizaron diluciones decimales consecutivas y se inocularon en placas $3 \mathrm{M}^{\mathrm{TM}}$ Petrifilm ${ }^{\mathrm{TM}}$ para el recuento rápido de Coliformes y E. coli ( $3 \mathrm{M}^{\mathrm{TM}}$, USA). Las colonias fueron enumeradas después de 18-24 h de incubación a $35 \pm 2{ }^{\circ} \mathrm{C}$ de acuerdo con las instrucciones del fabricante.

\section{Detección molecular de Salmonella}

Para detectar la presencia de Salmonella a partir de cada muestra recolectada se pesaron $325 \mathrm{~g}$ de carne molida de res en bolsa de polietileno tipo ziploc y se pre-enriquecieron en $975 \mathrm{~mL}$ de agua peptonada amortiguada ISO $\left(3 \mathrm{M}^{\mathrm{TM}}\right)$ atemperada a $41.5^{\circ} \mathrm{C}$. Cada muestra fue homogeneizada manualmente durante 1 min e incubada a $41.5^{\circ} \mathrm{C} \pm 0.5^{\circ} \mathrm{C}$ por un mínimo de $18 \mathrm{~h}$. Al término de la incubación cada muestra fue homogeneizada nuevamente de forma manual por 1 min y una alícuota de $20 \mu \mathrm{L}$ fue transferida a un tubo de solución de lisis del kit para la detección de Salmonella (MDA2SAL96, 3M Molecular Detection Assay 2-Salmonella) previamente atemperado a $100{ }^{\circ} \mathrm{C}$ por $30 \mathrm{~s}$ y mezclando 5 veces; los tubos se colocaron en un bloque térmico a $100^{\circ} \mathrm{C}$ por 15 min y luego se dejaron enfriar durante 5 min en el bloque a temperatura ambiente. De cada tubo se transfirieron $20 \mu \mathrm{L}$ a los microtubos de reacción y se mezcló 5 veces con la pipeta multicanal antes de colocarlos en el equipo de detección molecular 3M MDS (Loop Mediated Isothermal Amplification LAMPBioluminiscent Technolgy). Los resultados se interpretaron utilizando el software de detección molecular 3M específico para el equipo. Las muestras que resultaron positivas a la presencia de Salmonella fueron confirmadas por cultivo tradicional.

\section{Aislamiento e identificación de Salmonella}

El aislamiento e identificación de Salmonella se realizó de acuerdo al protocolo MLG 4.10 del Departamento de Agricultura de los Estados Unidos (USDA, 2019b). A partir del pre-enriquecimiento de cada muestra que resultó positiva por el método MDS se transfirió $1 \mathrm{~mL}$ a $10 \mathrm{~mL}$ de caldo de tetrationato (TT, NEOGEN, USA) y $0.1 \mathrm{~mL}$ a $10 \mathrm{~mL}$ de caldo Rappaport-Vassiliadis (RV, NEOGEN) para su enriquecimiento selectivo. Los caldos se incubaron en un baño de circulación termostático a $43 \pm 0.5^{\circ} \mathrm{C}$ y $42 \pm$ $0.5{ }^{\circ} \mathrm{C}$ por $24 \mathrm{~h}$, respectivamente. A partir de cada caldo se inocularon por estría placas con agar sulfito bismuto (SB, NEOGEN) y agar verde brillante sulfa (VBS, NEOGEN) y se incubaron a $35 \pm 2{ }^{\circ} \mathrm{C}$ por $24 \mathrm{~h}$. Las colonias típicas de Salmonella se confirmaron mediante pruebas bioquímicas en agar hierro y lisina (NEOGEN, USA), agar triple azúcar y hierro (NEOGEN) y caldo urea (DIFCO) incubadas a $35 \pm 2{ }^{\circ} \mathrm{C}$ por $24 \mathrm{~h}$. Las cepas atípicas fueron sometidas a pruebas bioquímicas adicionales en agar citrato de Simmons (NEOGEN, USA) y caldo MRVP (NEOGEN, USA). Los aislamientos que cumplieron el perfil típico o atípico fueron sometidos a prueba de aglutinación con antisuero Poly A-I \& Vi (DIFCO).

\section{Análisis de datos}

Los recuentos de coliformes totales y E. coli fueron expresados como unidades formadoras de colonia por gramo (UFC/g) y transformados a $\log _{10}(\log \mathrm{UFC} / \mathrm{g})$ previo a ser comparados utilizando un ANOVA ( $p<$ 0.05) para determinar si existían diferencias entre las muestras según el municipio de origen. La frecuencia de muestras positivas 
para Salmonella fue comparada por municipio utilizando la prueba de Chi cuadrada con un nivel de significancia $(p<$

\section{Resultados y Discusión}

Recuento de indicadores microbiológicos de higiene

El recuento de microorganismos indicadores permite evaluar las condiciones de higiene bajo las cuales se ha elaborado un alimento. En el caso de la carne molida, los niveles de coliformes y principalmente de E. coli en el producto son el resultado de la calidad microbiológica de la materia prima con la que fue elaborada, limpieza de todas las superficies que estuvieron en contacto
0.05). Los análisis se realizaron con el software SPSS Statistics ver 22.0.0.0 (IBM Corp., USA)

durante su fabricación y condiciones en que fue almacenada. Los niveles de coliformes totales en las 96 muestras analizadas oscilaron entre 2.2 y 7.4 logaritmos de unidades formadoras de colonia por gramo $(\log \mathrm{UFC} / \mathrm{g})$ con un promedio de $4.7 \pm 1.03$ $\log \mathrm{UFC/g}$, mientras que los recuentos de $E$. coli resultaron entre 1.7 y $6.9 \mathrm{log} \mathrm{UFC} / \mathrm{g}$ con un promedio de $3.5 \pm 0.77 \log \mathrm{UFC} / \mathrm{g}$ (Cuadro 1). Los recuentos promedio de ambos grupos indicadores no fueron significativamente diferentes $(p>0.05)$ en las muestras obtenidas de carnicerías de diferentes municipios.

Cuadro 1. Recuento de microorganismos indicadores de higiene en carne molida de res fabricada en carnicerías según el municipio de origen $(n=96)$

\begin{tabular}{|c|c|c|c|c|c|c|}
\hline \multirow{2}{*}{ Municipio } & \multicolumn{3}{|c|}{ Coliformes totales (log UFC/g) } & \multicolumn{3}{|c|}{ E. coli $(\log \mathrm{UFC} / \mathrm{g})$} \\
\hline & Media $\pm \mathrm{DS}^{a}$ & Min & Max & Media \pm DS & Min & Max \\
\hline A & $4.3 \pm 0.94 \mathrm{~A}^{b}$ & 2.2 & 6.7 & $3.4 \pm 0.67 \mathrm{~A}$ & 2.7 & 4.6 \\
\hline B & $4.8 \pm 1.16 \mathrm{~A}$ & 2.7 & 7.4 & $3.7 \pm 0.91 \mathrm{~A}$ & 2.7 & 6.8 \\
\hline $\mathrm{C}$ & $4.9 \pm 0.93 \mathrm{~A}$ & 2.3 & 6.8 & $3.4 \pm 0.69 \mathrm{~A}$ & 1.7 & 5.2 \\
\hline
\end{tabular}

${ }^{a} \mathrm{DS}=$ desviación estándar

${ }^{b}$ Valores seguidos de la misma letra no son significativamente diferentes $(p>0.05)$.

La normatividad de nuestro país establece estándares microbiológicos para la carne y productos cárnicos producidos en establecimientos de sacrificio y faenado mediante la Norma Oficial Mexicana NOM194-SSA1-2004; en esta Norma se establece un límite máximo permisible de 5,000 UFC de E. coli por gramo de carne molida refrigerada (3.7 log UFC/g). Estos límites no aplican para la carne molida almacenada en refrigeración y exhibida para su venta en los supermercados, debido a que el estándar evalúa las condiciones de higiene durante su preparación, y no condiciones del almacenamiento.
En el caso de las carnicerías incluidas en este estudio, se solicitó al carnicero que fabricara la carne molida en el momento de la visita de muestreo y no se recolectaron muestras de carne molida previamente elaborada $\mathrm{y}$ almacenada, precisamente con la finalidad de evaluar solo las condiciones de higiene durante la fabricación. El 38.5\% de las 96 muestras recolectadas en los tres municipios presentaron recuentos de $E$. coli que rebasaron el límite de 5,000 UFC/g (Cuadro 2). El Municipio B presentó el mayor porcentaje de muestras por arriba del límite $(\mathrm{n}=17)$, seguido de A $(\mathrm{n}=11)$ y $\mathrm{C}(\mathrm{n}=9)$. Solo el 24\% de las muestras presentaron recuentos $<500 \mathrm{UFC} / \mathrm{g}$ de E. coli, lo que contrasta con 
resultados encontrados en países como Argentina donde un programa de mejoras en las BPM en carnicerías trajo como resultado que el $66.3 \%$ de las muestras de carne molida de res analizadas presentaran recuentos $<500$ UFC/g (Leotta et al. 2016).

Cuadro 2. Niveles de microorganismos indicadores de higiene en carne molida de res fabricada en carnicerías en tres municipios de la Zona Metropolitana de Guadalajara (n=96)

\begin{tabular}{|c|c|c|c|c|c|}
\hline \multirow{2}{*}{$\begin{array}{l}\text { Recuento } \\
\text { (UFC/g) }\end{array}$} & \multicolumn{2}{|c|}{ Coliformes } & \multirow{2}{*}{$\begin{array}{l}\text { Recuento } \\
\text { (UFC/g) }\end{array}$} & \multicolumn{2}{|c|}{ E. coli } \\
\hline & No. muestras & $\%$ & & No. muestras & $\%$ \\
\hline$<10,000$ & 19 & 19.8 & $\leq 500$ & 23 & 24.0 \\
\hline $10,000-1^{\prime} 000,000$ & 69 & 71.9 & $1,000-4,000$ & 36 & 37.5 \\
\hline$>1^{\prime} 000,000$ & 8 & 8.3 & $\geq 5,000^{\mathrm{a}}$ & 37 & 38.5 \\
\hline Total & 96 & 100 & Total & 96 & 100 \\
\hline
\end{tabular}

ámite máximo permitido de E. coli en carne molida refrigerada en establecimientos de sacrificio y faenado (NOM-194-SSA1-2004)

Para el caso de los coliformes totales no existen estándares microbiológicos, sin embargo, los resultados muestran que el $8.3 \%$ de las muestras presentaron valores superiores a 1'000,000 UFC/g; el municipio $\mathrm{B}$ presentó el mayor número de muestras con valores de coliformes > $6.0 \mathrm{log} \mathrm{UFC} / \mathrm{g}$. Los coliformes son un grupo de bacterias Gram negativas susceptibles a tratamientos térmicos y al efecto de agentes sanitizantes, por lo que suelen usarse como indicadores de higiene e indicadores de la eficacia de procesos de limpieza y sanitización de equipos y superficies (Cabrera-Diaz et al. 2013). En un estudio realizado en la ciudad de Monterrey se encontraron niveles de coliformes > $6.0 \mathrm{log} \mathrm{UFC} / \mathrm{g}$ en el $43 \%$ de muestras de carne molida colectadas en supermercados y carnicerías (Heredia et al. 2001).

Los elevados recuentos de indicadores microbiológicos relacionados con condiciones de higiene señalan que en el $38.5 \%$ de las 96 carnicerías estudiadas, existen fallos a las BPM en la preparación de carne molida de res, lo que genera un producto de mala calidad microbiológica, ya sea por una deficiente limpieza y desinfección de equipos, utensilios y superficies utilizadas en su fabricación, y/o por la alta carga microbiológica de los recortes de carne utilizados como materia prima para su elaboración. En una investigación previa realizada en 65 carnicerías de la Zona Metropolitana de Guadalajara, también se reportaron recuentos elevados de $E$. coli en carne molida de res con un promedio de $4.5 \pm 1.2 \mathrm{log} \mathrm{UFC} / \mathrm{g}$ y un rango de 2.5 a 7.6 log UFC/g (MartínezChávez et al. 2015); en dicha investigación se documentó que las principales fallas a las BPM observadas fueron el abuso de temperatura de la carne utilizada como materia prima, la sobrecarga de refrigeradores donde se almacenaba la materia prima, uso de tablas para cortar la materia prima con grietas visibles, falta de limpieza y sanitización apropiada de utensilios y falta de limpieza del molino con la frecuencia apropiada. Estas mismas fallas a las BPM se observaron en el presente estudio, siendo la limpieza inadecuada del molino una falla frecuente observada por los inspectores oficiales de los ayuntamientos participantes 
quienes estuvieron a cargo de las visitas de muestreo.

Para la producción de carne molida a nivel industrial, el Comité Internacional de Especificaciones Microbiológicas Alimentos (ICMSF, por sus siglas en inglés) recomienda verificar la higiene del proceso a través del recuento de $E$. coli en lotes de producto terminado aplicando un plan de muestreo de tres clases donde se recolecten 5 muestras por lote, de las cuales sólo se permite que 3 de ellas contengan recuentos de $E$. coli entre 10 y $100 \mathrm{UFC} / \mathrm{g}$ y ninguna muestra debe exceder $100 \mathrm{UFC} / \mathrm{g}$ (ICMSF 2011). Estos recuentos tan bajos son posibles cuando la carne molida se prepara a nivel industrial utilizando recortes de carne producida en rastros donde se aplican además de BPM, múltiples intervenciones antimicrobianas en la carne; si bien no son alcanzables ni aplicables en carnicerías, nos permiten reiterar que la producción de carne molida de res con bajas cargas microbianas es posible y que los establecimientos pequeños pueden mejorar sus prácticas de higiene para obtener productos de mejor calidad microbiológica e inocuidad, y que $E$. coli es un indicador útil para verificar la higiene del proceso. Sin embargo, antes de implementar cualquier programa de muestreo microbiológico, se debe reforzar la capacitación del personal de carnicerías y supervisar la aplicación de acciones concretas que mejoren las BMP en estos establecimientos.

Los lineamientos generales de higiene que deben seguir los establecimientos productores de alimentos, entre los cuales se incluyen las carnicerías, están descritos en la Norma Oficial Mexicana NOM-251-SSA12009, la cual establece los requisitos mínimos de buenas prácticas de higiene que deben observarse en el proceso de alimentos y sus materias primas a fin de evitar su contaminación a lo largo de su proceso.
Adicionalmente, organizaciones como Canada Beef (Canada Beef 2020) recomiendan prácticas de higiene específicas para la fabricación de la carne molida de res que incluyen mantener la temperatura de los recortes de carne utilizados como materia prima a un máximo de $-2{ }^{\circ} \mathrm{C}$, mantener la temperatura del área donde se procese la carne en $10^{\circ} \mathrm{C}$ como máximo; limpiar y desinfectar el molino y utensilios antes de utilizarlos, y asegurarse de que el personal cumpla con las buenas prácticas de higiene; lavar y sanitizar el molino al menos 2 veces al día, y al terminar el turno de trabajo realizar un lavado completo del mismo separando las piezas (cedazos, navajas, gusano, volante, cabezal y charola); nunca guardar el cabezal sucio ni dejar recortes de carne en la charola o cedazo; y con respecto a la materia prima, utilizar recortes de carne con bajas cargas microbiológicas procedentes de establecimientos de sacrificio certificados, almacenarse en refrigeración y protegidos de la contaminación. Si bien, estas recomendaciones están orientadas a la industria cárnica de gran escala, pueden adaptarse para ser alcanzables en la fabricación de carne molida en carnicerías de pequeña escala como las incluidas en este estudio.

Por otro lado, se ha demostrado que la aplicación de tratamientos antimicrobianos a base de ácido peroxiacético o de ácido sulfúrico/sulfato de sodio, en los recortes utilizados como materia prima permite reducir la diversidad de microoganismos y abundancia de enterobacterias en carne molida (Weionroth et al. 2019), lo que puede mejorar la calidad microbiológica del producto y su vida de anaquel, sin embargo, es importante recalcar que antes de considerar la aplicación de cualquier tratamiento químico para reducir la carga microbiana en la materia prima, se debe asegurar el cumplimiento de las BPM. 
En Argentina por ejemplo, se demostró que la implementación de un programa de mejora de las BPM en carnicerías y de un programa de capacitación de empleados basado en un diagnóstico de problemas de cada establecimiento, redujo significativamente la contaminación de la carne molida de res y de las superficies, equipos y utensilios de las carnicerías (Leotta et al. 2016); programas de este tipo podrían implementarse en países como el nuestro donde una gran proporción de la carne molida se fabrica en carnicerías locales.

\section{Detección de Salmonella}

Salmonella se detectó en 66 de las 96 muestras analizadas $(68.8 \%)$ por el método molecular MDS; el aislamiento del patógeno fue confirmado por cultivo en 64 de las 66 muestras. En dos muestras no se recuperaron cepas típicas o atípicas de Salmonella debido probablemente a que fueron inhibidas por la microbiota asociada, o bien a que se trataba de cepas sumamente atípicas que no cumplieron con los criterios de identificación bioquímica y serológica empleados. La frecuencia de aislamiento por municipio de origen de las muestras fue del $23.9 \%$ para los municipios A y B, y del $21 \%$ para el municipio C (Figura 1). No se encontró diferencia en la frecuencia de muestras positivas a Salmonella por municipio de procedencia $(p>0.05)$.

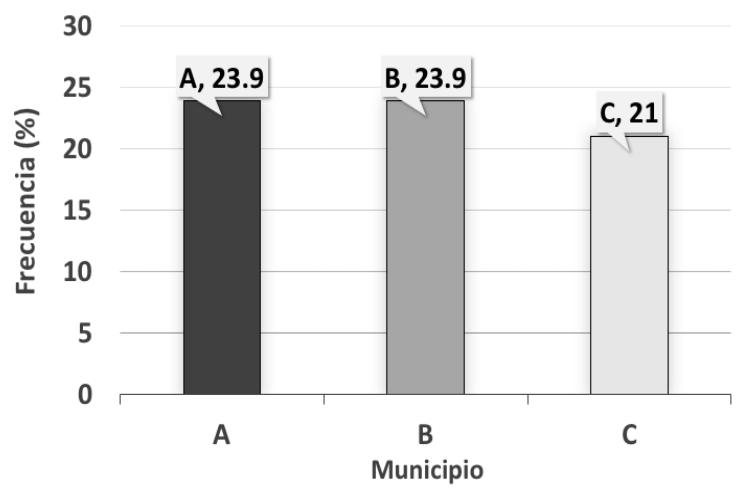

Figura 1. Frecuencia de aislamiento de Salmonella en carne molida de res fabricada en carnicerías de tres municipios de la Zona Metropolitana de Guadalajara $(\mathrm{n}=96)$

Estos resultados son semejantes a los reportados por nuestro grupo en dos investigaciones previas realizadas en carnicerías de la Zona Metropolitana de Guadalajara, donde Salmonella fue detectada en el $56.7 \%$ de 238 muestras y en el $71 \%$ de 65 muestras de carne molida de res analizadas (Cabrera-Diaz et al. 2013; Martínez-Chávez et al. 2015) y coinciden también en las fallas a las BMP observadas. Estos resultados sugieren que, a lo largo de los años, las condiciones de higiene de este tipo de establecimientos no han mejorado significativamente $y$ no se ha logrado reducir la alta frecuencia de Salmonella en este producto. Por otra parte, la frecuencia observada de Salmonella fue mayor que la reportada en un estudio realizado en Monterrey, donde el patógeno fue encontrado en el $11.4 \%$ de 88 muestras de carne molida de res de supermercados y carnicerías (Heredia et al. 2001) y también fue mayor que la encontrada en la Ciudad de México, donde el patógeno fue aislado del $32 \%$ de 50 muestras colectadas en carnicerías, tianguis y mercados públicos (Nayarit-Ballesteros et al. 2016) y del $11.1 \%$ de 864 muestras colectadas en mercados sobre ruedas (VillalpandoGuzmán et al. 2017); en ambos estudios, Salmonella fue aislada con una frecuencia significativamente menor a partir de muestras de carne molida de res colectadas 
de supermercados. Con respecto a estudios de otros países, la frecuencia observada de Salmonella en el presente estudio fue considerablemente mayor; en Egipto el patógeno fue aislado del $26.7 \%$ de 90 muestras de carne molida de res colectada en carnicerías y supermercados (Sallam et al. 2014), en Turquía del $24 \%$ de 50 muestras de carnicerías (Siriken et al. 2020), en Argentina del $10.4 \%$ de 86 muestras de carnicerías (Leotta et al. 2016) en Irlanda del 3\% de 100 muestras de supermercados (Khen et al. 2014), y en los EUA sólo del $0.66 \%$ de 2,199 muestras colectadas en tiendas al menudeo en 38 ciudades (Vipham et al. 2012), y del 3.8\% de 1,750 muestras colectadas en tiendas al menudeo en la ciudad de Seattle, Washington (Samadpour et al. 2006); el muestreo de verificación oficial del Departamento de Agricultura de ese país reportó en el año 2019 una prevalencia nacional de Salmonella del $2.2 \%$ a partir de 10,932 muestras de carne molida de res de establecimientos de todo el país (USDA 2020). Es importante destacar que en los EUA, el $91 \%$ del volumen total de carne molida de res que se fabrica, se hace a nivel industrial en grandes establecimientos y la baja prevalencia del patógeno refleja condiciones de producción en donde se cuenta de forma obligatoria con BPM y con la implementación del Sistema de Análisis de Peligros y Puntos de Control Crítico (HACCP, por sus siglas en inglés) que suele incluir intervenciones antimicrobianas en los recortes de carne usados como materia prima. Actualmente está por implementarse un nuevo estándar de desempeño en ese país, con el que se pretende reducir el porcentaje de muestras positivas a Salmonella permitidas en carne molida de res fabricada en grandes establecimientos a fin de reducir la prevalencia nacional del patógeno y con ello, los casos de salmonelosis asociados al consumo de este producto (USDA 2019a).
Como se ha mencionado, el recuento de $E$. coli es utilizado como un indicador del control del proceso para evitar la contaminación de la carne y productos cárnicos durante su producción; al respecto, se observó que de las 66 muestras que resultaron positivas a la presencia de Salmonella, 15 (22.7\%) presentaron recuentos de $E$. coli $\leq 500 \mathrm{UFC/g}$ mientras que $51(77.3 \%)$ contenían más de 1,000 UFC/g (Figura 2). De las 66 muestras, 24 $(36.3 \%)$ contenían niveles $\geq 5,000 \mathrm{UFC} / \mathrm{g}$ que corresponde al límite normativo en nuestro país para carne molida refrigerada para determinar las condiciones de higiene durante su producción. Si bien, no existe una correlación directa entre la presencia de E. coli y la presencia de patógenos, los resultados indican que el mayor número de muestras positivas a Salmonella se presentó en aquellas muestras con $\geq 1,000 \mathrm{E}$. coli/g. Las fallas en las BPM durante la fabricación de carne molida de res no solo conducen a obtener un producto con alta carga microbiológica cuya vida de anaquel se verá reducida de manera significativa mermando la calidad del producto, también representan una mayor probabilidad de contaminación por patógenos que pondrán en riesgo la salud de los consumidores. $\mathrm{Si}$ bien, la carne molida está destinada a cocinarse completamente antes de consumirse, el mal manejo durante su preparación en las cocinas de los consumidores puede generar el consumo del producto insuficientemente cocinado, o puede dar lugar a contaminación cruzada, transfiriendo los patógenos hacia alimentos listos para consumo, poniendo en riesgo la salud de los consumidores. En algunos casos, existen recetas culinarias como la "carne apache" típica de Michoacán, preparada con carne molida de res cruda marinada en jugo de limón y adicionada de jitomate, cebolla y cilantro (también conocida como "ceviche de carne molida"), 
que evidentemente representan un riesgo a la salud de los consumidores. Se ha demostrado que el marinado con jugo de limón que se hace en la preparación de ceviche de pescado no logra destruir Salmonella y Vibrio cholerae debido a que su actividad antimicrobiana es neutralizada por las proteínas (Mathur \& Schaffner 2013; Torres Vitela et al. 2000).

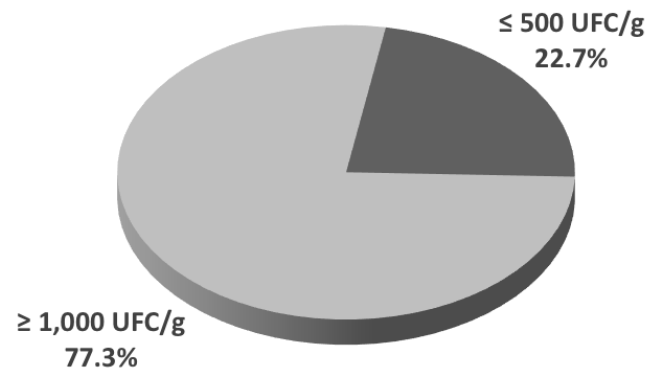

Figura 2. Porcentaje de muestras de carne molida de res positivas a Salmonella según el recuento de E. coli que presentaron $(\mathrm{n}=96)$

Finalmente, los resultados de este estudio han sido presentados a inspectores de los Ayuntamientos participantes para enfatizar la importancia de la vigilancia en el cumplimiento de la normatividad con respecto a las prácticas de higiene en el proceso de producción de alimentos (NOM-251-SSA1-2009) y se recomienda que sean utilizados en los cursos de capacitación en manejo higiénico de alimentos y BPM que se impartan tanto a inspectores como a propietarios $y$ trabajadores de carnicerías.

\section{Conclusiones}

La falta de cumplimiento de las BPM en carnicerías de tres municipios de la Zona Metropolitana de Guadalajara resultó en la fabricación de carne molida de res con altas cargas microbianas y alta frecuencia de Salmonella, lo que conlleva un riesgo potencial para el consumidor. Es necesario mejorar la capacitación en BPM del personal que labora en carnicerías, así como intensificar la inspección oficial que obligue su cumplimiento por parte de las autoridades correspondientes.

\section{Agradecimientos}

Los autores agradecen a la Dra. Delia Guillermina González Aguilar y al M.C. Carlos Pacheco Gallardot por su apoyo en la realización del proyecto. Esta investigación fue desarrollado con fondos del Programa de Apoyo a la Mejora en las Condiciones de Producción SNI (PROSNI 2019) de la Universidad de Guadalajara.

\section{Referencias}

Canada Beef. Buenas prácticas en el manejo de la carne de res molida. Disponible desde Internet en <http://www.canadabeef.mx/portfolio -item/buenas-practicas-en-el-manejode-carne-de-res-molida/> [Consultado 14 Junio 2020].

Cabrera Díaz, E., J. Varela Hernández, C.A. Campos Bravo \& A. Castillo. 2010. Inocuidad de carnes rojas. pp. 129-179. En: Bianchi, G. y O.D. Feed. Introducción a la Ciencia de la Carne. Editorial Hemisferio Sur. Buenos Aires, Argentina. [ISBN 978-9974674-20-2].

Cabrera-Diaz, E., C.M. Barbosa-Cardenas, J.A. Pérez-Montano, D.G. GonzálezAguilar, C. Pacheco-Gallardo, \& J. Barba. 2013. Occurrence, serotype diversity, and antimicrobial resistance of Salmonella in ground beef at retail stores in Jalisco state, Mexico. Journal of Food Protection 76:2004-2010.

CDC [Centers for Disease Control and Prevention]. 2018. Surveillance for foodborne disease outbreaks-United States, 2009-2015. MMWR 67:1-16. 
CDC [Centers for Disease Control and Prevention]. 2019. Outbreak of Salmonella infections linked to ground beef. Disponible desde Internet enhttps://www.cdc.gov/salmonella/du blin-11-19/index.html [Consultado 01 Junio 2020].

CDC [Centers for Disease Control and Prevention]. 2020. Foodborne Disease Outbreak Surveillance System. Disponible desde Internet en: $<$ https://www.cdc.gov/fdoss/publicati ons.html> [Consultado 01 Junio 2020]. Clinical and Laboratory Standards Institute. 2008. Performance standards for antimicrobial susceptibility testing; 18th informational supplement, 8th ed. Approved standard M100- S18.

Diaz, L., Li, Y., Kubota, R. y Jenkins, D. 2019. Characterization of a portable, non-instrumented incubator for enrichment of Escherichia coli O157:H7 and Salmonella serovar Typhimurium and detection by loop mediated isothermal amplification (LAMP). Food Protection Trends 39:40-50.

Heredia, N., S. Garcia, G. Rojas \& L. Salazar. 2001. Microbiological condition of ground meat retailed in Monterrey, Mexico. Journal of Food Protection 64:1249-1251.

Hernández-San Juan, J.S., A. ZúñigaEstrada, I. Sánchez-Ortega, J. CastroRosas, A.D. Román-Gutiérrez \& E.M. Santos-López. 2007. Microbiological conditions during the slaughter process at a municipal slaughterhouse in Hidalgo, Mexico. Veterinaria México 38:187-195.

ICMSF [International Commission on Microbiological Specifications for Foods]. 2011. Microorganisms in Foods 8, Use of data for assesing process control and product acceptance. Springer, NY, USA. [ISBN 978-1-4419-9373-1].
Khen, B.K., O.A. Lynch, J. Carroll, D.A. McDowell \& G. Duffy. 2014. Prevalence and characteristics of Salmonella in the beef chain in the Republic of Ireland. Zoonoses and Public Health 61:534-536.

Leotta, G.A., V. Brusa, L. Galli, C. Adriani, L. Linares, A. Etcheverría, M. Sanz, A. Sucari, P. Peral García \& M. Signorin. 2016. Comprehensive evaluation and implementation of improvement actions in butcher shops. PLoS One 11:e0162635.

Martínez-Chávez, L., E. Cabrera-Diaz, J.A. Pérez-Montano, L.E. Garay-Martínez, J.J. Varela- Hernández, A. Castillo, L. Lucia, M.G. Ávila-Novoa, M.A. Cardona-López, P. GutiérrezGonzález \& N.E. Martínez-Gonzáles. 2015. Quantitative distribution of Salmonella spp. and Escherichia coli on beef carcasses and raw beef at retail establishments. International Journal of Food Microbiology 210:149-155.

Mathur, P. \& D.W. Schaffner. 2013. Effect of lime juice on Vibrio parahaemolyticus and Salmonella enterica inactivation during the preparation of the raw fish dish ceviche. Journal of Food Protection 76:1027-1030.

Narvaez-Bravo, C., Miller, M.F., Jackson, T., Jackson, S., Rodas-Gonzalez, A., Pond, K., Echeverry, A., Brashears, M.M., 2013. Salmonella and Escherichia coli $\mathrm{O} 157: \mathrm{H} 7$ prevalence in cattle and on carcasses in a vertically integrated feedlot and harvest plant in Mexico. Journal of Food Protection 76:786-795.

Nayarit-Ballesteros N., M.S. RubioLozano, E. Delgado-Suárez, D. Méndez-Medina, D. Braña, D. Varela \& C. Rodas-Suárez. 2016. Perfil de resistencia a antibióticos de serotipos de Salmonella spp. aislados de carne 
de res molida en la Ciudad de México. Salud Pública de México 58:371-377.

Perez-Montaño, J.A., D.G. Gonzalez

Aguilar, J. Barba, C. PachecoGallardo, C.A. Campos-Bravo, S. Garcia, N.L. Heredia \& E. CabreraDiaz. 2012. Frequency and antimicrobial resistance of Salmonella serotypes on beef carcasses at small abattoirs in Jalisco State, Mexico. Journal of Food Protection 75:867873.

Ruiz, H., M.F. Miller, S. Gragg, G.H. Loneragan, L.G. Garcia \& M.M. Brashears. 2013. Salmonella prevalence in beef lymph nodes and feces from cattle harvested at five Mexican abattoirs. Meat Science 96:489-490.

Sallam K.I., M.A. Mohammed, M.A. Hassan \& T. Tamura. 2014. Prevalence, molecular identification and antimicrobial resistance profile of Salmonella serovars isolated from retail beef products in Mansoura, Egypt. Food Control 38:209-214.

Samadpour M., M.W. Barbour, T. Nguyen, T.M. Cao, F. Buck, G.A Depavia, E. Mazengia, P. Yang, D. Alfi, M. Lopes, J.D. Stopforth. 2006. Incidence of enterohemorrhagic Escherichia coli, Escherichia coli O157, Salmonella, and Listeria monocytogenes in retail fresh ground beef, sprouts, and mushrooms. Journal of Food Protection 69:441-3.

Secretaría de Salud. 2019. Compendio de anuarios de morbilidad 1984-2018. Disponible desde Internet en: <http://187.191.75.115/anuario/html/a nuarios.html> [Consultado 18 Junio 2020].

Siriken B., Al G. \& I. Erol. 2020. Prevalence and antibiotic resistance of salmonella enteritidis and Salmonella Typhimurium in ground beef and meatball samples in Samsun, Turkey.
Microbial Drug Resistance 26:136144.

Torres-Vitela, M.R., A. Castillo, L.M. Ibarra-Velazquez, V. NavarroHidalgo, M.O. Rodríguez-García, N.E. Martínez-Gonzáles \& J.A. PérezMontaño. 2000. Survival of Vibrio cholerae $\mathrm{O} 1$ in ceviche and its reduction by heat pretreatment of raw ingredients. Journal of Food Protection 63:445-450.

USDA [United States Department of Agriculture]. 2019a. Changes to the Salmonella verification testing program: proposed performance standards for Salmonella in raw ground beef and beef manufacturing trimmings and related agency verification procedure. Federal Register 84:57688-57695.

USDA [United States Department of Agriculture]. 2019b. Isolation and identification of Salmonella from meat, poultry, pasteurized egg, and siluriformes (fish) products and carcass and environmental sponges. MLG 4.10. Disponible desde Internet en:

<https://www.fsis.usda.gov/wps/wcm/ connect/700c05fe-06a2-492a-a6e13357f7701f52/MLG4.pdf?MOD=AJPERES $>$ [Consultado 02 Junio 2020].

USDA [United States Department of Agriculture]. 2020. Sampling results for FSIS regulated products. Disponible desde Internet en: $<$ https://www.fsis.usda.gov/wps/wcm/ connect/68f5f6f2-9863-41a5-a5c425cc6470c09f/Sampling_Project_Res ults_Data.pdf?MOD=AJPERES $>$

[Consultado 18 Junio 2020].

Villalpando-Guzmán, S., C.R. VázquezQuiñones, I. Natividad-Bonifacio, E. Curiel-Quesada, E.I. QuiñonesRamírez y C. Vázquez-Salinas. 2017. Frecuencia, susceptibilidad 
antimicrobiana y patrón de adherencia de Salmonella enterica aislada de carne de pollo, res y cerdo de la Ciudad de México. Revista Chilena de Infectología 34:458-466.

Vipham J.L., M.M. Brashears, G.H. Loneragan, A. Echeverry, J.C. Brooks, W.E. Chaney \& M.F. Miller. 2012. Salmonella and Campylobacter baseline in retail ground beef and whole-muscle cuts purchased during 2010 in the United States. Journal of Food Protection 75:2110-2115.

Weinroth, M.D., B.C. Britton, K.R. McCullough, J.N. Martin, I.
Geornaras, R. Knight, K.E. Belk \& J.L. Metcalf. 2019. Ground beef microbiome changes with antimicrobial decontamination interventions and product storage. PLOS ONE 14: 0217947.

Zaidi, M. B., J.J. Calva, M.T. EstradaGarcía, V. León, G. Vázquez, G. Figueroa, E. López, J. Contreras, J. Abbott, S. Zhao, P. McDermott \& L. Tollefson. 2008. Integrated food chain surveillance system for Salmonella spp. in Mexico. Emerging and Infectious Diseases 14: 429-435. 